\title{
QUALIDADE DE CAJUS-DE-MESA OBTIDOS NOS SISTEMAS DE PRODUÇÃO INTEGRADA E CONVENCIONAL ${ }^{1}$
}

\author{
ANA PAULA SILVADE ANDRADE², VITOR HUGO DE OLIVEIRA ${ }^{3}$, RENATO INNECCO $^{4}$, EBENÉZER DE OLIVEIRA SILVA $^{5}$
}

RESUMO - O cajueiro (Anacardium occidentale L.) é uma planta de grande importância econômica para o Nordeste brasileiro, pela diversidade de produtos proporcionados pelo fruto e pedúnculo e pela quantidade de empregos gerados. Apesar disso, inexiste uma padronização nos sistemas de produção empregados, com reflexos negativos na produção e qualidade da matéria-prima destinada ao consumo in natura e à indústria. A conversão dos sistemas de produção vigentes para o sistema de produção integrada poderá contribuir para atenuar esse quadro. O objetivo deste trabalho foi comparar os sistemas de produção integrada e convencional para cajueiro-anão precoce quanto à qualidade do pedúnculo. $\mathrm{O}$ experimento foi instalado em um pomar comercial, localizado no município de Beberibe (CE), numa área de aproximadamente 1,0 ha, onde foram desenvolvidos os sistemas de Produção Integrada (PI) e Convencional (PC). Cada um ocupou uma área de 0,5 ha, separados entre si por uma bordadura composta de cinco fileiras de plantas. No sistema PI, foram aplicadas as práticas recomendadas nas Normas Técnicas de Produção Integrada de Caju. No PC, foram aplicadas as práticas comumente utilizadas pelo produtor. Foram avaliados cor da película, firmeza de polpa, sólidos solúveis totais (SST), acidez total titulável (AT), teor de vitamina C e pH. Para essas variáveis, foram estimadas médias a partir das 12 amostras obtidas nos dois tratamentos, que foram comparadas, utilizando-se do teste $\mathrm{t}(\mathrm{P} \leq 0,05)$. Verificou-se, para as variáveis AT e teor de vitamina $\mathrm{C}$, que as médias obtidas no sistema PI foram significativamente superiores às do PC. Quanto ao pH, observou-se diferença significativa entre as médias obtidas nos sistemas, sendo o valor obtido no PC superior ao do PI. Os cajus produzidos no sistema de produção integrada apresentaram melhor qualidade.

Termos para indexação: Anacardium occidentale L., produção integrada de frutas, qualidade pós-colheita.

\section{POST-HARVEST QUALITY OF THE CASHEW APPLES GOTTEN IN THE INTEGRATED FRUIT PRODUCTION AND THE CONVENTIONAL CROPPING SYSTEMS}

ABSTRACT - The cashew tree (Anacardium occidentale L.) is a plant of great importance for the Brazilian Northeast Region, due to the diversity of products generated for the fruit and peduncle and the amount of generated jobs. Despite this, there is no standardization in the cropping systems presently used, with negative consequences in the yield and quality of the raw material for consumption and for industry. The conversion of traditional orchards to the integrated fruit production systems will contribute to minimize this scenery. The objective of this work was to compare the integrated fruit production (IFP) and conventional cropping production (CP) systems in dwarf cashew orchard, as to apple quality. The experiment was installed in a commercial orchard, located in Beberibe Country, State of Ceará, Brazil, in an area of 1.0 hectare, where the IFP and CP systems had been developed. Each system had occupied 0.5 hectare, separated by five crop lines of plants. In the IFP system, cropping practices were applied according to the Legal Marks of Integrated Fruit Production in Brazil. In CP system, cropping practices were applied accordingly with common used by local growers. Apple color, firmness, soluble solids (SS), titratable acidity (TA), vitamin $\mathrm{C}$ content and $\mathrm{pH}$ were evaluated. For these variables, means were estimated from twelve samples obtained in both treatments, which were compared by test $\mathrm{t}(\mathrm{P} \leq 0.05)$. For the $\mathrm{TA}$ and vitamin $\mathrm{C}$ content variable, the IFP system was significantly superior to the $\mathrm{CP}$ ones. For the $\mathrm{pH}$ variable, a significant difference was observed between the averages of the systems, being the value gotten in the CP superior to the one of the IFP. The cashew apples obtained in the IFP system showed better quality. The post-harvest quality of the cashew apples had been influenced by the production systems. Index terms: Anacardium occidentale L., integrated fruit production, post-harvest quality.

\section{INTRODUÇÃO}

O cajueiro (Anacardium occidentale L.) é uma planta de grande importância econômica, pois o fruto e pedúnculo podem originar produtos variados. Também tem relevante importância socioeconômica para o País, uma vez que a exploração de aproximadamente 690.131 hectares de cajueiros mobilizam no campo 300 mil pessoas e proporcionam uma produção anual de
147.129 t de castanha (IBGE, 2006) e 1.650 .000 t de pedúnculo (FAO, 2006).

Apesar dessa pujança e do enorme potencial, o agronegócio caju atravessa uma crise agrícola e econômica, com implicações na produção e na geração de emprego e renda, frutos da monocultura predatória, sem foco de mercado, ocupando extensas áreas, em detrimento do uso de tecnologias apropriadas. As práticas de desmatamento sem manejo florestal, queimadas e

'(Trabalho 076-07). Recebido em: 23-03-2007. Aceito para publicação em: 20-07-2007. Projeto realizado com apoio financeiro do MAPA/CNPq, Embrapa Agroindústria Tropical e UFC. Parte integrante da Dissertação de Mestrado em Fitotecnia.

${ }^{2}$ Eng $^{\mathrm{a}}$ Agra MSc. E-mail: anapaulasandrade@gmail.com.

${ }^{3}$ Eng $^{\circ}$ Agr $^{\circ}$ DSc. Pesquisador A - Embrapa Agroindústria Tropical. E-mail: vitor@enpat.embrapa.br

${ }^{4}$ Eng $^{\circ}$ Agr $^{\circ}$ DSc. Professor titular do Departamento de Fitotecnia/UFC. E-mail: innecco@ufc.br.

${ }^{5}$ Eng $^{\circ}$ Agr $^{\circ}$ DSc. Pesquisador A - Embrapa Agroindústria Tropical. E-mail: bene@enpat.embrapa.br.

Rev. Bras. Frutic., Jaboticabal - SP, v. 30, n. 1, p.176-179, Março 2008 
uso de áreas impróprias para o cultivo, contribuem para o baixo rendimento, degradação ambiental e distanciamento das demandas por alimentos produzidos fora dos moldes tradicionais (OLIVEIRA et al., 1997). Com isto, a cadeia produtiva do caju brasileiro, a partir da metade da década de oitenta, vem apresentando sinais evidentes de perda de competitividade (OLIVEIRA\& ANDRADE, 2004).

Além disso, os mercados mundiais passaram a exigir a qualidade externa das frutas e o controle sobre todo o sistema de produção, incluindo a análise de resíduos nos frutos e estudo sobre o impacto ambiental, para realizarem suas importações (FACHINELLO et al., 2000).

A conversão de pomares tradicionais para o sistema de Produção Integrada de Frutas (PIF), que consiste na obtenção de frutos de alta qualidade, minimizando o uso de agroquímicos e preservando o meio ambiente, a saúde do consumidor e do produtor, poderá contribuir para a reversão desse quadro. Este trabalho teve o objetivo de comparar os sistemas de Produção Integrada e Convencional para cajueiro-anão precoce, quanto à qualidade do pedúnculo, na safra de 2005-2006, em Beberibe (CE).

\section{MATERIAL E MÉTODOS}

O trabalho foi realizado durante um ciclo agrícola (20052006), num pomar comercial de cajueiro com quatro anos de idade, conduzido em espaçamento $7 \times 7 \mathrm{~m}$, localizado no município de Beberibe (CE), cujas coordenadas geográficas são: latitude de $04^{\circ} 13^{\prime} 15^{\prime \prime} \mathrm{S}$, longitude de $38^{\circ} 12^{\prime} 34^{\prime \prime}$ W Grm e altitude de $55 \mathrm{~m}$. Foram utilizadas mudas do tipo anão precoce, sendo o clone CCP 09 o enxerto e o CCP 06 o porta-enxerto. O experimento foi instalado numa área de aproximadamente um hectare, onde foram desenvolvidos os sistemas de Produção Integrada (PI) e Convencional (PC). Cada um ocupou uma área de 0,5 ha, separados entre si por uma bordadura composta de cinco fileiras de plantas. O solo da área experimental apresentava textura arenosa e características físicas e químicas homogêneas.

No sistema PI, aplicaram-se as práticas recomendadas pelas Normas Técnicas Específicas para a Produção Integrada de Caju (NTEPICaju), segundo Oliveira (2003), que consistiram da manutenção, nas entrelinhas, de cobertura vegetal viva (vegetação nativa) com aproximadamente cinco centímetros de altura; limpeza das linhas de plantio, a uma profundidade máxima de cinco centímetros; adubação, realizada conforme análise do solo, que recomendou a aplicação de $200 \mathrm{~g}$ de N planta $^{-1}$ ano $^{-1}$, de $180 \mathrm{~g}$ de $\mathrm{K}_{2} \mathrm{O}$ planta $^{-1}$ ano $^{-1}$, que foram parcelados em doses mensais, via fertirrigação, e de $300 \mathrm{~g}$ de $\mathrm{P}_{2} \mathrm{O}_{5}$ planta $^{-1}$ ano $^{-1}$, aplicado diretamente no solo em dose única; irrigação do tipo microaspersão individual por planta, que aplicava, no período de uma hora e meia, o volume de $78 \mathrm{~L}_{\text {planta }}{ }^{-1}$ dia $^{-1}$; manejo da parte aérea realizado por meio de três podas, sendo uma de limpeza, no final da safra, uma de manutenção, no início do período chuvoso, e outra para levantamento da copa a uma altura de $50 \mathrm{~cm}$ acima do solo e controle fitossanitário, realizado com base em sistema de amostragem, segundo Mesquita et al. (2002) e Cardoso \& Freire (2002), realizando-se uma aplicação com fungicida à base de oxicloreto de cobre $(6,5 \mathrm{~g} / \mathrm{L})$. Nesse sistema, a colheita foi feita manualmente, em horários com temperaturas amenas. Do total de cajus colhidos nas 12 plantas, retirou-se uma amostra de 50 cajus para análise da qualidade. Posteriormente, foram acondicionados em caixas plásticas, com capacidade de $15 \mathrm{~kg}$, numa única camada, contendo uma esponja de um centímetro de espessura no fundo.

Para a condução no sistema PC, aplicaram-se as práticas comumente utilizadas pelo produtor, que consistiram de limpeza das entrelinhas, mediante capina, a uma profundidade de $15 \mathrm{~cm}$, e limpeza das linhas, a uma profundidade máxima de cinco centímetros. O método de irrigação e as recomendações de adubação do solo foram similares às empregadas no sistema PI. O manejo da parte aérea consistiu de apenas uma poda de limpeza, realizada no final da safra, e os tratamentos fitossanitários foram aplicados, sem o auxílio de sistemas de amostragens, no início do período de produção e após cada fluxo produtivo, constituindo-se de três aplicações de fungicida à base de oxicloreto de cobre $(6,5 \mathrm{~g} / \mathrm{L})$ e uma com inseticida à base de fenitrothion $(150 \mathrm{ml} / 100 \mathrm{~L})$. Nesse sistema, a colheita foi realizada acondicionando-se os cajus em caixas similares, mas sem camada de esponja no fundo e em mais de uma camada. Do total de cajus colhidos nas 12 plantas, retirou-se uma amostra de 50 cajus para análise da qualidade.

Em ambos os sistemas, os cajus foram colhidos quando se desprendiam facilmente da panícula, por meio de uma leve torção com as pontas dos dedos, evitando-se, assim, o contato direto com a mão do colhedor. Em seguida, os cajus colhidos foram transportados para o Laboratório de Fisiologia e Tecnologia Pós-Colheita da Embrapa Agroindústria Tropical e caracterizados fisicamente quanto à cor da película, através de duas leituras realizadas nos pedúnculos, utilizando-se de colorímetro da marca Minolta, modelo CR 300, e firmeza de polpa, através de duas leituras realizadas na porção apical do pedúnculo, utilizando-se de penetrômetro manual, modelo FT 011, com ponteiras de $8 \mathrm{~mm}$ de diâmetro. Após as avaliações físicas, foram congelados em freezer doméstico, a aproximadamente $-20^{\circ} \mathrm{C}$, e posteriormente descastanhados.

Os pedúnculos foram cortados em quatro pedaços iguais, no sentido longitudinal, e distribuídos em quatro sacos diferentes (um pedaço em cada saco). Em seguida, foram analisadas as seguintes variáveis: acidez titulável (AT) (\%), determinados através de titulação com solução de $\mathrm{NaOH}(0,1 \mathrm{~N})$; sólidos solúveis totais (SST), determinados através do refratômetro digital, da marca ATAGO PR-101 ( ${ }^{\circ}$ Brix); vitamina C (mg/100g), determinada por titulometria com solução de DFI (2,6 diclorofenolindofenol $0,02 \%$ ) e $\mathrm{pH}$, medido diretamente no suco, utilizando-se de potenciômetro com membrana de vidro. Para essas variáveis, foram estimadas médias a partir das amostras obtidas nos dois tratamentos, que foram comparadas, utilizandose o teste $\mathrm{t}$, adotando-se o nível de significância de 5\%.

\section{RESULTADOS E DISCUSSÃO}

Avaliando-se as características firmeza de polpa, sólidos solúveis totais e cor, observou-se que os resultados obtidos, em 
ambos os sistemas, não apresentaram diferença significativa.

Quanto à variável AT, observou-se diferença significativa nos resultados obtidos entre os sistemas PI e PC, onde a média obtida no primeiro sistema foi superior à do segundo (Figura 1). Esse fato pode ter ocorrido devido às diferentes técnicas póscolheita empregadas. Pois a técnica empregada no sistema PC influencia negativamente no teor de vitamina $\mathrm{C}$, resultando num valor de AT inferior, quando comparado com o do PI.

O resultado obtido para essa característica, no sistema PC, foi semelhante ao encontrado por FIGUEIREDO (2000), que, estudando o clone CCP 76 , obteve $0,29 \%$ de acidez no estádio de maturação comercial. No entanto, o valor encontrado neste trabalho foi superior aos encontrados por SILVA JÚNIOR \& PAIVA(1994) e AGOSTINE-COSTA et al. (2004), que, estudando as características de pedúnculos do clone CCP 09, em área de produção convencional e sob sequeiro, relataram valores médios de AT de $0,23 \%$ e $0,25 \%$ em clones de Pacajus, e $0,24 \%$ em clones de Paraipaba, respectivamente. Tais diferenças, quando comparadas com os resultados obtidos neste trabalho, podem ser atribuídas às diversas condições edafoclimáticas dos locais de realização dos experimentos, bem como de manejo e idade das plantas avaliadas.

As médias de $\mathrm{pH}$ obtidas de pedúnculos, nos dois sistemas analisados, foram estatisticamente diferentes, onde o valor obtido no sistema PC foi superior ao do sistema PI (Figura 2). Esse resultado não é comumente encontrado na literatura, mas pode ter ocorrido devido à significativa diferença encontrada entre as médias do teor de ácidos orgânicos nos sistemas.

De acordo com KAYS (1991), o pH é influenciado diretamente pela concentração de ácidos orgânicos, que é representada pela AT. Os resultados encontrados para essa característica, no sistema PC, ficaram acima dos observados por SILVA JÚNIOR \& PAIVA (1994) e PAIVA et al. (1998), que, estudando pedúnculos do clone CCP 09, obtiveram valores médios de, respectivamente, 4,31 e 4,1. Contudo, avaliando clones de cajueiro-anão precoce, MOURA (2004) relata que, normalmente, a variação do $\mathrm{pH}$ nessa espécie é de 4,10 a 4,64 e, corroborando esta afirmação, AGOSTINE-COSTA et al. (2004), avaliando a mesma característica em pedúnculos do clone CCP 09, oriundos de Pacajus (CE) e Paraipaba (CE), encontraram, respectivamente, valores de 4,36 e 4,41. Embora a diferença entre os valores de $\mathrm{pH}$ tenha sido significativa, é considerada pequena e encontra-se dentro da faixa de variação acima definida.

$\mathrm{O}$ valor obtido para o teor de vitamina C no sistema PI diferiu estatisticamente e foi superior ao obtido no sistema PC (Figura 3). Essa diferença pode ter ocorrido devido às melhores práticas pós-colheita na PI, quando comparadas às práticas na PC, uma vez que a vitamina $\mathrm{C}$ apresenta alta sensibilidade aos fatores do meio e outros fatores intrínsecos do processo de senescência (KLEIN, 1987; FAVELL, 1998). Esses fatores podem ter contribuído, em conjunto, para a diferença entre as médias dos teores de vitamina $\mathrm{C}$.

O teor de vitamina C obtido no sistema $\mathrm{PC}$ foi inferior aos relatados por SILVAJÚNIOR \& PAIVA(1994) e PAIVA et al. (1998), que, avaliando as características dos pedúnculos do clone CCP 09, em área de produção convencional, obtiveram, respectivamente, 170,00 e 160,34mg/100g de vitamina C. Também foi inferior aos valores obtidos por SOUZA FILHO (1987), que, realizando o mesmo estudo, em clones CCP 76, 1001 e 06, obteve, respectivamente, $158 ; 26 ; 157,60$ e $153,20 \mathrm{mg} / 100 \mathrm{~g}$ de vitamina $\mathrm{C}$. As diferenças entre os teores encontrados por esses autores e os obtidos neste experimento podem ser atribuídas à localização dos plantios, aos diferentes tratos culturais e aos tipos de solo.

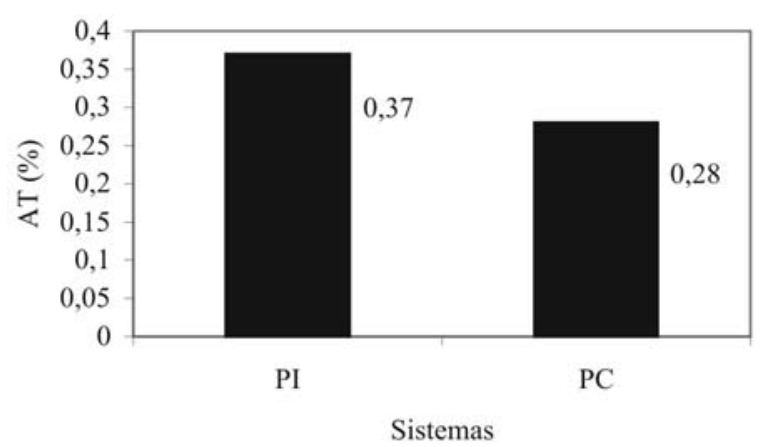

FIGURA 1 - Avaliação da acidez total titulável em pedúnculos de cajueiro-anão precoce, conduzidos sob o sistema PI e PC. Beberibe - CE, safra 2005-2006.

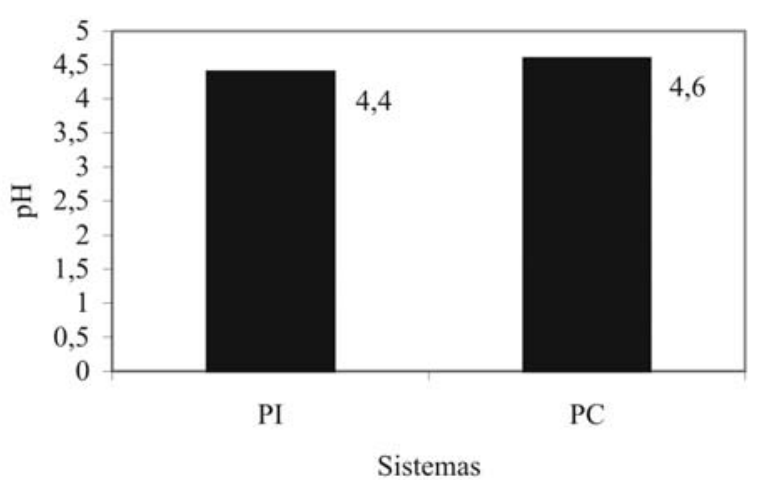

FIGURA 2 - Avaliação do $\mathrm{pH}$ em pedúnculos de cajueiro-anão precoce, conduzidos sob o sistema PI e PC. Beberibe - CE, safra 2005-2006.

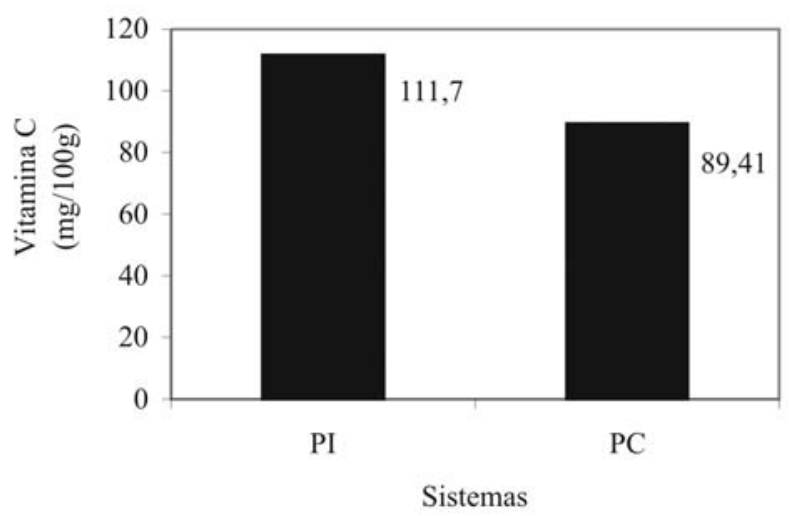

FIGURA 3 - Avaliação do teor de vitamina $\mathrm{C}$ em pedúnculos de cajueiro-anão precoce, conduzidos sob o sistema PI e PC. Beberibe - CE, safra 2005-2006. 


\section{CONCLUSÃO}

Os cajus produzidos no sistema de produção integrada apresentaram melhor qualidade.

\section{REFERÊNCIAS}

AGOSTINE-COSTA, T. da SILVEIRA; JALES, K.A.; GARRUT, D. S.; PADILHA, V.A.; LIMA, J.B. de; AGUIAR, M.J.; PAIVA, J.R. de. Teores de ácido anacárdico em pedúnculos de cajueiro Anacardium microcarpum e em oito clones de Anacardium occidentale var. nanum disponíveis no Nordeste do Brasil. Ciência Rural, Santa Maria, v. 34, n. 4, p. 1075-1080, 2004.

CARDOSO, J.E.; FREIRE, F.C.O. Identificação e manejo das principais doenças. In: MELO, Q.M.S. (Org.). Caju: Fitossanidade. Brasília: Embrapa Informação Tecnológica, 2002. p.41-51. (Frutas do Brasil, 26).

FACHINELLO, J.C.; GRUTZMACHER, A.D.; HERTER, F.G.; CANTILLANO, F.; MATTOS, M.L.T.; FORTES, J.F.; AFONSO, A.P.S.; TIBOLA, C.S. Avaliação do sistema de produção integrada de pêssego de conserva na região de Pelotas - safra 1999-2000. In: SEMINÁRIO DE PRODUÇÃO INTEGRADA DE FRUTAS, 2., 2000, Bento Gonçalves. Resumos... Bento Gonçalves: Embrapa Uva e Vinho, 2000. p.78-84.

FAO - FAOSTAT. Disponível em: <http://faostat.fao.org/site/ 567/desktopdefault.aspx?pageid=567>. Acesso em: $17 \mathrm{fev}$. 2006.

FAVELL, D.J. A comparison of the vitamin C content of fresh and frozen vegetables. Food Chemistry, Kidlington, v.62, n.1, p.5964. 1998.

FIGUEIREDO, R.W. Desenvolvimento, maturação e armazenamento de pedúnculos de cajueiro-anão precoce CCP 76 sob influência do cálcio. 2000. 149 f. Tese (Doutorado em Ciência dos Alimentos) - Faculdade de Ciências Farmacêuticas, Universidade de São Paulo, 2000.

IBGE - Banco de dados agregados. Disponível em: w w w . s i d r a i b g e.gov.br/b d a / t a b e la/ listabl.asp? $\mathrm{z}=\mathrm{t} \& \mathrm{o}=18 \& \mathrm{i}=\mathrm{p} \& \mathrm{c}=1618>$. Acesso em: 10 fev. 2006.

KAYS, J.S. Postharvest physiology of perishable plant products. New York: AVI Book, 1991.543p.
KLEIN, B.P. Nutritional consequences of minimal processing of fruits and vegetables. Journal of Food Quality, Trumbull, v. 10, p. 179-193, 1987.

MESQUITA, A.L.M.; BRAGA SOBRINHO, R.; OLIVEIRA, V.H. Monitoramento de pragas na cultura do cajueiro. Fortaleza: Embrapa Agroindústria Tropical, 2002. 36p. (Embrapa Agroindústria Tropical. Documentos, 48).

MOURA, C.F.H. Armazenamento de pedúnculos de cajueiroanão precoce BRS 189, CCP 76, END 189 sob diferentes temperaturas e atmosferas. 2004. 181f. Tese (Doutorado em Fitotecnia)-Centro de Ciências Agrárias, Universidade Federal do Ceará, Fortaleza, 2004.

OLIVEIRA, V.H.; ANDRADE, A.P.S.; Produção integrada melhora a qualidade do caju. Revista Agroanalysis, São Paulo, v. 24, n. 5, p. 28-29, 2004.

OLIVEIRA, V.H. Normas técnicas e documentos de acompanhamento da produção integrada de caju. Fortaleza: Embrapa Agroindústria Tropical, 2003. 75p. ( Documentos, 66).

OLIVEIRA, V.H.; SANTOS, F.J.S.; MIRANDA, F.R.; ALMEIDA, J.I.L.; SAUNDERS, L.C.U. Avanços de pesquisa em cajucultura irrigada. In: WORKSHOP AVANÇOS DE PESQUISA EM AGROINDÚSTRIA TROPICAL, 1, 1997, Fortaleza. Palestras... Fortaleza: Embrapa Agroindústria Tropical, 1997. p. 26-27.0

PAIVA, J. R.;ALVES, R. E.; BARROS, L.M.; CAVALCANTI, J.J.V.; ALMEIDA, J.H.S.; MOURA, C.F.H. Produção e qualidade de pedúnculos de clones de cajueiro-anão-precoce sob cultivo irrigado. Fortaleza: Embrapa Agroindústria Tropical, 1998. 5p. (Comunicado Técnico, 19).

SILVA JÚNIOR, A.; PAIVA, F.F.A. Estudos físico e físico-químico de clones de cajueiro-anão precoce. Fortaleza: EPACE, 1994. 19p. (Boletim de pesquisa, 23).

SOUZA FILHO, M. de S. M. de. Aspectos da avaliação física, química, físico-química e aproveitamento industrial de diferentes clones de caju (Anacardium occidentale L.). 1987. 167f. Dissertação (Mestrado em Fitotecnia) - Centro de Ciências Agrárias, Universidade Federal do Ceará, Fortaleza, 1987. 